\title{
L'APPRENTISSAGE DE LA REDACTION À L'EPREUVE DES MANUELS SCOLAIRES
}

\author{
A APRENDIZAGEM DA REDAÇÃO À PROVA DOS LIVROS DIDÁTICOS
}

\section{THE CONTRIBUTION OF TEXTBOOKS IN THE LEARNING OF WRITING}

\author{
Sylvie Plane* \\ Université Paris-Sorbonne, Paris, França \\ Kathy Similowski** \\ Université Paris-Sorbonne, Paris, França
}

Résumé: La pratique de la rédaction dès le début de la scolarité se met en place en France officiellement à partir de 1880. Toutefois, son enseignement ne va pas s'imposer sans difficulté. En effet, les programmes donnent des directives parfois contradictoires et les procédures mises en place par les enseignants se révèlent partiellement inefficaces si bien que les résultats sont estimés trop faibles par I'Institution. Dans la bataille des idées qui conduit à une évolution des textes réglementaires mais aussi à des pratiques divergentes, quel a été le rôle des manuels? Notre contribution se propose d'alimenter la réflexion sur les fonctions et la place des manuels dans l'enseignement de la rédaction à travers la comparaison de trois manuels scolaires à usage dans les classes de fin d'école primaire (cours moyen) au cours de la période allant de l'après-guerre jusqu'au « Plan de rénovation de l'enseignement du français » $(1971)$. Ces trois ouvrages $(1947,1956,1969)$ révèlent, à travers leurs interprétations des programmes, des influences sociales et idéologiques contrastées. Ils renseignent sur les interrogations qui traversent la communauté éducative sur le statut des savoirs, les modes d'enseignement et d'apprentissage et les finalités de l'école.

Mots-clés: Écriture; Rédaction; Manuels scolaires; École primaire française; Méthodes.

Resumo: A prática da redação desde o início da escolaridade tem espaço na França oficialmente a partir de 1880. Contudo, seu ensino não se impôs sem dificuldades. Na verdade, os programas dão diretrizes às vezes contraditórias e os procedimentos utilizados pelos professores se revelam parcialmente ineficazes, assim como os resultados esperados pela Instituição são muito fracos. Na batalha das ideias que conduzem a uma evolução dos textos regulamentais, mas também às práticas divergentes, qual a funç̃õo dos livros didáticos? Nossa contribuição se propõe a alimentar a reflexão sobre as funções e o lugar dos livros didáticos nas salas do final da escola primária (curso médio) ao longo do período que se estende do pós-guerra até o "Plano de renovação do ensino francês" (1971). Essas três obras $(1947,1956,1969)$ revelam, através das respectivas interpretações dos programás, as influências sociais e ideológicas constrantantes. Eles informam sobre as interrogacões que atravessam a comunidade educativa sobre o status dos saberes, os modos de ensino e de aprendizagem, bem como as finalidades da escola.

Palavras-chave: Escrita; Redação; Livro didático; Escola primária francesa; Métodos.

* Professeure émérite à l'Université Paris-Sorbonne, laboratoire EA 4509 Sens Texte Informatique Histoire, Vice-présidente du Conseil Supérieur des Programmes de l'Éducation Nationale ; sylvie.plane@wanadoo.fr

** Doctorante à l'Université Paris-Sorbonne, Paris, France; ESPE de l'Académie de Versailles, Université de Cergy-Pontoise, France; chercheur au sein du laboratoire EA 4509, Équipe « dynamique de la production discursive », Université Paris-Sorbonne, Paris, France; kathy.similowski@orange.fr 


\begin{abstract}
The policy consisting in teaching structured writing from the very beginning of school has been officially established in France in the early 1880s. However, this policy did not prevail without difficulties. Indeed, official programs have at times given contradictory guidelines and the procedures established by the teachers have proven partly ineffective. As a consequence, results have been considered insufficient by the Institution. What was the role of textbooks in the debate that made official instructions evolve while generating divergent practices in the field? Our contribution aims at fostering ideas on the institutional function and on the practical role of textbooks in the teaching of writing. To this end, we compare three textbooks in use in primary school late classes during the aftermath of World War II and until the advent of the "Renovation Plan for the Teaching of French" (1971). These three books $(1947,1956,1969)$ reveal contrasting social and ideological influences, through different interpretations of the official progams. They provide information on the questioning that animate the educational community regarding the status of knowledges, teaching methods, learning dynamics, and the finalities of school.
\end{abstract}

Keywords: Writing; Redaction; Textbooks; French Primary School; Methods.

\title{
Introdução
}

L'Ecole d'autrefois sépare les enfants des classes populaires et les enfants des classes moyennes ou supérieures. Les programmes et les méthodes pédagogiques sont marqués par cette inégalité institutionnellement encouragée. Seule l'école primaire a vocation à apprendre à tous. Outil de communication et vecteur de culture, le français à l'école primaire occupe une place privilégiée et son enseignement est fondamentalement tourné vers l'écrit. La maîtrise de la langue passe d'abord par celle de l'écriture au sens graphique du terme, de l'orthographe et de la grammaire. L'accès aux œuvres littéraires et la rédaction de phrases simples « dès le début de la scolarité » se mettent en place officiellement à partir de 1880. Mais l'enseignement de la « rédaction $»^{1}$ va devoir surmonter bien des obstacles avant de s'imposer. Il se heurte d'abord au faible degré d'instruction des maitres, pour lesquels l'Institution engagera des réformes successives afin d'élever leur niveau de formation. Ensuite, si la composition de petits textes est présentée comme l'objectif ultime de "l'unité d'enseignement » du français, les instituteurs répugnent à lui consacrer la place qu'elle mérite, lui préférant la dictée, dont Ferdinand Buisson² ne parvient pas à débarrasser le Certificat d'Études ${ }^{3}$. Surtout, il faut aux pédagogues chercher un autre modèle que celui porté par le Secondaire ${ }^{4}$ qui suit un schéma bien tracé datant d'une époque révolue, celle de l'hégémonie du latin, où la tradition rhétorique, l'exercice de la version, le contact fréquent avec les textes classiques et leur

1 Jules Ferry (promoteur de l'école gratuite et obligatoire) impose le terme, mais l'exercice va recevoir encore diverses appellations tout au long du XXe siècle.

2 Ferdinand Buisson est directeur de l'Enseignement Primaire en France de 1879 à 1896.

3 Le Certificat d'Études ou Certificat d'Études Primaires Élémentaires (1866) était un diplôme sanctionnant la fin de l'enseignement élémentaire en France et attestant l'acquisition des connaissances de base lécriture, lecture, calcula, histoire-géographie, sciences). Il a été officiellement supprimé en 1989.

4 Enseignement qui se situe après la fin de l'école primaire et avant l'enseignement supérieur. 
imprégnation, sont considérés comme un passage obligé avant la composition. Des questions fondamentales interrogent le législateur et la communauté éducative: faut-il laisser écrire les écoliers et comment ? Ont-ils les capacités suffisantes pour développer des habilités rédactionnelles ? Que faut-il leur enseigner pour répondre aux exigences de la vie pratique et les préparer à leurs destinées professionnelles ? Les tensions qui parcourent l'enseignement de la rédaction ne sont pas que didactiques et tiennent finalement aux missions que l'on veut prêter à l'école. Que se joue-t-il en effet à travers cet enseignement ? Quelque part, si comme le disent les instructions officielles, l'écrit a pour vocation d'éveiller les élèves à la pensée ${ }^{5}$, alors c'est aussi de l'émancipation des classes populaires dont il est question.

A travers l'enseignement de la rédaction, on peut observer toutes les tensions. Celle qui traverse d'abord la didactique avec ses deux tendances : l'une, conforme aux traditions, qui s'oriente vers l'imitation de textes. Elle offre à l'élève comme à l'instituteur le cadre rassurant du modèle, mais favorise la reproduction de mots au détriment peut être de la pensée. L'autre axée sur l'observation du réel, de la vie quotidienne, scolaire ou familiale dont l'enfant est censé rendre compte avec précision et sincérité tandis que son imagination simultanément est sollicitée, en apparence du moins, à travers des exercices dits d'invention. L'enseignement de la rédaction réinterroge les certitudes pédagogiques encore vivantes du début du XXe siècle et notamment celles qui postulent que la lecture et la parfaite connaissance de la langue doivent précéder l'écriture et que l'écolier n'a pas besoin d'écrire plus que des phrases simples et correctes. Dès la fin du XIXe siècle et dans les années qui suivent, l'enseignement de la rédaction à l'école primaire est considéré comme un échec. Force est d'observer que les programmes donnent des directives parfois contradictoires (imitation des grands auteurs ou invention? Compte rendu d'observation du réel ou expression personnelle ? Enseignement pratique ou formation de la pensée ?). Les procédures mises en place par les enseignants se révèlent partiellement inefficaces et les résultats sont estimés trop faibles par l'Institution ${ }^{6}$; « Au Certificat d'Études, c'est l'épreuve la plus faible ». (BISHOP, 2005)

Notre contribution se propose d'alimenter la réflexion sur les fonctions et la place des manuels dans l'enseignement de la rédaction sous un angle historique. A partir des années 1880 , les enseignants se sont vus confier le libre choix de leurs livres de classe avec pour seule restriction que ce choix s'exerce collectivement au sein d'une

5 « La composition française est destinée non seulement à enseigner à écrire, mais encore, mais surtout à enseigner à penser » (Félix Pécaut, Directeur des études de l'École Normale Supérieur des Institutrices de Fontenay, Revue de l'enseignement primaire, 24 juin 1891 p 371-372)

6 Jean Zay, Ministre de l'Éducation Nationale, confirme encore en 1938 « Les résultats de l'enseignement de la composition française à l'école primaire sont assez décevants. 
même école. Ils deviennent les prescripteurs de leur outil d'enseignement et de l'outil d'apprentissage de leurs élèves. (PLANE, 1999, p. 18)

Dans la bataille des idées qui conduit à une évolution des textes réglementaires mais aussi à des pratiques divergentes, quel a été le rôle des manuels? Quelles influences peut-on retrouver des injonctions institutionnelles et des résistances ou des avancées pédagogiques ? Comment ont-ils pu guider les maîtres et aider les élèves?

Puisque « l'idéal d'une scolarisation totale n'est devenu une réalité qu'après la Deuxième Guerre mondiale » (CHERVEL, 1995), nous avons choisi d'analyser et de comparer au cours de la période allant de l'après-guerre (1945) jusqu'au « Plan de Rénovation de l'Enseignement du Français » (1971), trois manuels scolaires largement diffusés et en usage dans les classes de fin d'école primaire (Cours Moyen) :

- «Doux parler de France, Lecture Langue Française, Cours Moyen » (PIERRE ANTOINE, 1947)

- «Rédigeons, le résumé de lecture, le vocabulaire, le paragraphe par l'observation. Cours Moyen »(PALMERO Jean; FELIX Alexis, 1956)

- « Méthode active d'initiation à la composition française par le vocabulaire et la construction de phrases » (GALICHET, René; DUPLOUY, Fernand, 1969).

\section{Les attentes institutionnelles confrontées à une interprétation classique des programmes}

Ces trois ouvrages prennent place dans les écoles française après la publication d'Instructions parues pendant la seconde guerre mondiale ${ }^{8}$. Au Cours Moyen', les élèves sont conviés à résumer des récits lus ou étudiés en classe. On les invite à reproduire les tournures intéressantes. Sont recommandés des exercices simples sur des sujets d'observation empruntés à la vie réelle, aux activités scolaires, professionnelles, familiales, nationales, à propos desquels l'enfant exprime ce qu'il voit, sent ou imagine. D'autres travaux à caractère pratique sont également conseillés

7 Le « Plan Rouchette » publié en janvier 1971 donnera une nouvelle définition des finalités de l'enseignement du français et notamment de l'enseignement élémentaire et se substituera à toutes les instructions antérieures. Il s'agira notamment de tenir compte de la création d'un premier cycle du second degré (1959) que fréquentent tous les enfants jusqu'à 16 ans.

8 Instructions relatives au Nouveau Plan d'Études des Écoles Primaires du 5 mars 1942.

9 Dans le système éducatif français, le cours moyen (deux ans) désigne sous la troisième République le cours intermédiaire entre le cours élémentaire et le cours supérieur (cours complémentaire à l'école primaire supprimé en 1959). Il clôt officiellement (encore aujourd'hui) les cours de l'enseignement primaire (maternelle puis élémentaire). 
comme des lettres, compte rendus, des procès-verbaux ou des rapports. L'ambition, dit-on, n'est pas « le style » mais de permettre aux écoliers de s'exprimer simplement et sobrement en un langage direct, « de traduire avec exactitude, netteté et précision les données multiples de l'expérience et de l'observation ». L'époque qui sépare l'après-guerre de l'après mai 1968 fourmille de textes réglementaires, mais le triptyque préconisé est toujours le même résumer, reproduire, rédiger des récits tirés de l'expérience et de l'observation. Il s'agit d'apprendre à ordonner et relier des phrases simples, correctes et précises en un paragraphe. Le Certificat d'Études qui sanctionne la fin de l'école primaire prévoit « une rédaction sur un sujet simple se rapportant à la vie personnelle de l'enfant» (Arrêté du 22 février 1946), mais ce sujet peut être aussi, dans un souci de formation à la vie professionnelle et sociale, un compte rendu, un rapport, ou une lettre (Arrêté du 28 octobre 1947).

Si l'on met de côté les écrits à caractère pratique, l'Institution s'oriente donc vers des sujets qui permettent à l'élève de produire un écrit grâce à l'observation $d u$ réel et le rappel de son vécu personnel. Et cet enseignement doit servir non seulement à savoir écrire mais aussi à former la pensée. Car à travers de petits exercices "d'invention » et de rédaction, lélève doit s’habituer « à exprimer sa pensée » (Arrêté du 17 octobre 1945) ou « à préciser sa pensée et à s'exprimer avec plus de clarté et de correction. »(Circulaire du 19 octobre 1960) ${ }^{10}$

Les hésitations pédagogiques de la période sont révélées par une circulaire du 17 mars 1952 relative aux conférences pédagogiques portant sur les exercices de rédaction. Elle précise en effet que:

les instructions officielles sur l'enseignement du français ont signalé divers exercices: expression libre, compte rendu, résumé d'exercices d'observation d'histoire et de géographie, résumé et compte rendu de lecture, imitation, conversion, construction de phrases, reproduction de textes, compte rendu d'enquête, mise en forme d'un raisonnement, rédaction collective en équipe, composition française.

Malgré le caractère polysémique de certaines termes, on constate que les pratiques oscillent entre plusieurs tendances opposées: celle de l'imitation (reproduction de textes) et de l'invention ${ }^{11}$ (expression libre), de la lecture (résumé et compte

10 La circulaire concernant l'enseignement du français et du calcul dans les classes primaires du 19 octobre 1960 rappelle que les élèves doivent être capables d'ordonner leurs idées dans un petit paragraphe. L'entraînement est le gage de la réussite : " « Ils n'y parviendront qu'au prix de répétitions fréquentes et d'exercices nombreux. ». "C'est en multipliant les exercices d'analyse, d'élocution et de rédaction qu'on habituera l'enfant à préciser sa pensée et à s'exprimer avec plus de clarté et de correction. 》

11 L'Arrêté concernant les horaires et programmes des écoles primaires du 17 octobre 1945 prévoit aux Cours Moyens de petits exercices d'invention et de construction de phrases de types variés, des exercices 
rendu de lecture) et de l'écriture, de la phrase (construction de phrases) au texte, de la langue outil scolaire (résumé d'exercices d'observation d'histoire et de géographie) social ${ }^{12}$ (compte rendu) ou objet littéraire (composition française).

Les trois manuels révèlent également leurs interprétations des programmes. Le premier (1947) rédigé par deux inspecteurs d'académie Pierre Besseige et Antoine Lyonnet sous le pseudonyme Pierre Antoine offre une interprétation classique des exigences institutionnelles ${ }^{13}$. Le ton est donné dans la préface. Il ne s'agit pas d'innover mais de rester fidèle à une méthode « qui depuis vingt ans a rencontré la faveur constante du personnel et des élèves et qu'on n'a pas réussi à détrôner parce qu'elle est la plus directe, la plus naturelle et la plus efficace ». Cette méthode, c'est l'observation des textes "éprouvés, des textes classiques ». Il s'agit de donc de rédiger une phrase ou un paragraphe par imitation. Et alors que le législateur met les instituteurs en garde contre cette tentation, l'ouvrage incite à la recherche du beau style. (Ex: «Faisons de jolies phrases » p.184). La «préparation à la composition française » qui constitue la partie de la « rédaction " (même si le terme nest pas repris) n'est encore qu'un appendice de l'enseignement du «Vocabulaire » et de l' « Élocution ».

Jean Palmero, directeur d'école normale, et Alexis Felix, instituteur de classe d'application, proposent un manuel (1956) qui s'inscrit entre respect des préconisations réglementaires et poids des traditions. La première partie du manuel, de loin la plus importante, privilégie en effet l'apprentissage de la rédaction par l'observation du réel. A l'époque des «leçons de choses ${ }^{14}$, cette observation est guidée par un questionnaire donnant lieu à la rédaction d'une «fiche d'observation ». En fin de « leçon » lélève rédige un « cahier de travaux libres » dont un exemple est donné au début de l'ouvrage. Ce cahier se veut un espace de liberté au sein duquel l'élève

très simples de rédaction, habituant l'enfant à exprimer sa pensée en phrases ordonnées et bien liées. L'Arrêté fixant le programme des classes de fin d'études du 25 juillet 1947 ouvre la possibilité de " sujets libres » à côté des « sujets empruntés à la vie (vie familiale, vie scolaire, vie pratique) » et des comptes rendus de lecture. Les Instructions sur le Certificat d'Études Primaires du 30 octobre 1947 préconisent deux sujets : "Un des deux sujets pourrait convenir surtout aux esprits positifs (rapport, compte rendu, PV, lettre d'affaires) et l'autre surtout aux imaginatifs et aux sensibles (lettre, récit) »

12 La circulaire sur l'organisation du Certificat d'Études Primaires du 16 avril 1949 recommande d'éviter des sujets pratiques " ceux qui présentent un caractère trop spécial, qui font appel à une expérience d'adulte et qui déroutent les candidats »

13 Ce manuel s'inscrit dans la continuité d'un autre ouvrage d'avant-guerre des mêmes auteurs intitulé "Lecture et Langue française, Cours Moyen ( $2^{\mathrm{e}}$ degré) préparation au certificat d'études Primaire » (21 e édition en 1935)

14 La « leçon de choses » est un principe éducatif théorisé à la fin du XIXe siècle et consistant à partir d'un objet concret pour faire acquérir à l'élève une idée abstraite. 
" reste libre d'organiser à son goût sa mise en page ». ${ }^{15}$ Entre « la fiche d'observation » et le "cahier de travaux libres », un déroulement est minutieusement décrit valable pour chaque leçon. Le point d'arrivée et l'objectif annoncé de l'ouvrage c'est bien la rédaction. Pour " moderne » qu'il puisse apparaître ce manuel n'opère pas pour autant une rupture catégorique avec la démarche classique qui s'appuie sur l'étude de la langue et sur les textes littéraires. Si la "fiche d'observation » permet d'amorcer un travail sur le "Vocabulaire » et d'engager des « conversations " avec les élèves («Élocution»), un texte d'auteur d'une quinzaine de lignes est proposé pour compléter « le travail du style » (la recherche du «style » est donc toujours présente). Un nouveau texte court (6 lignes environ) intitulé « Bel exemple » doit servir de guide: « $\grave{A}$ votre tour en vous inspirant de ce bel exemple et en vous souvenant de vos observations, écrivez un court et précis instantané sur un ou plusieurs des sujets suivants...» (p. 5)

On peut donc dire que ce manuel semble conforme aux prescriptions institutionnelles en tant que la rédaction est annoncée comme un objectif d'apprentissage autonome. C'est ce que les auteurs déclarent dans leur préface et communiquent à travers le titre de l'ouvrage ${ }^{16}$. Par ailleurs, l'élève semble bien invité à observer le réel pour produire (fiche d'observation, cahier de travaux libres). Mais cette observation se greffe sur une démarche qui reste classique parce que l'étude du vocabulaire y est encore prépondérante, la recherche du style présente et que l'imitation des « beaux textes » vient parfaire la démarche personnelle de l'élève. Quant à la seconde partie du manuel, elle est résolument tournée vers les textes à travers l'exercice du « résumé de lecture ». La démarche est au service de la compréhension littéraire autant qu'à l'écriture. La rédaction est indissociable là encore de la recherche de style et de l'imitation avec une partie « Rédigeons » comprenant des exercices qui « attirent l'attention de l'écolier sur les procédés élémentaires de style, dont il ne saurait se passer pas plus qu'il ne pourrait spontanément les découvrir » et « lui montrent ce qu'il faut faire autant que ce qu'il en faut pas faire » et « le contraignent à ne jamais s'éloigner du texte ».

René Galichet, Professeur de Collège et Fernand Duplouy, Directeur d'École offrent une méthode plus active (1969), amorce d'un tournant sans doute dû à la «massification » de l'enseignement. Car dans ce manuel, la « leçon de composition française » est clairement identifiée et clôt chaque chapitre ${ }^{17}$. L'objectif des auteurs

15 On y suggère : dessins, copies de textes d'auteur, collages de documents (ex : gravure de paysages au printemps p. 105), listes (de fleurs par exemple p 105), descriptions (de la prairie au printemps par exemple p. 105), courts écrits comme des compte rendus d'enquête...

16 «Rédigeons, le résumé de lecture, le vocabulaire, le paragraphe par l'observation. Cours Moyen »

17 Les auteurs partent d'un constat : « De nos jours, l'enseignement du français est de plus en plus morcelé ». 
est de susciter le « goût et le désir d'écrire ». L’expérience et l'observation personnelle de l'élève restent convoquées, et ce conformément aux programmes. En effet, la leçon de composition française s'ouvre sur une partie " préparation à la rédaction " où l'élève est sollicité par différents procédés: recherches lexicales, observations, enquêtes, débats, développement de son opinion. En voici des exemples:

Cherchez la signification précise du mot « artisan » dans votre dictionnaire (p. 52) Fabriquez un petit album de feuilles mortes, et dessinez celle qui vous semble la plus belle. (p. 28)

Quels fruits récolte-t-on en automne dans votre région ? Renseignez-vous sur les différentes espèces. (p. 34)

En classe, ouvrez un débat sur les avantages et les inconvénients comparés du cinéma et de la télévision. (p. 132)

Parmi les vedettes du chant, laquelle admirez-vous le plus ? Dites pourquoi ? (p. 144)

Le texte a perdu son rôle de modèle absolu. S'il reste présent pour être lu, expliqué et observé, l'extrait d'auteur n'est plus le point de départ d'un processus de pure imitation par reproduction. Il est désacralisé et observé pour identifier les mécanismes de composition ou pour être manipulé:

Réécrivez à votre manière la fin du récit de Marcel Pagnol (« Nous descendions... ») en changeant l'ordre du récit : vous commencerez par expliquer la « toilette » ensuite, vous décrirez l'installation. (p. 11)

Ecrivez une autre conclusion pouvant convenir au sujet d'étude. (p. 47)

Par ailleurs, lecture et écriture sont dissociées: le résumé de lecture ne fait pas l'objet d'un traitement particulier. C'est un point, parmi d'autres, traité dans la partie méthodologie. Car ce qui caractérise cet ouvrage c'est l'accent mis sur les points méthodologiques suffisamment nombreux pour constituer l'une des trois parties de la « leçon de composition française ».

Ils souhaitent redonner à cet enseignement " une réelle unité, une solide cohérence. » Après la lecture et le vocabulaire intervient une "leçon de construction de phrases", sorte de "questionnaire méthodique auquel les élèves auront à répondre le plus souvent oralement, guidés par leur maître» et qui sert de transition avec la rédaction. 


\section{Des influences sociales et idéologiques très présentes}

Le premier ouvrage (1947) s'inscrit dans la période d'après-guerre et s'affuble d'une mission patriotique, civique et morale: «Après les années sombres que nous venons de vivre, il nous a paru que nous devions mettre l'accent sur la Patrie ». Le manuel prend en charge l'éducation de l'élève en contribuant à «sa formation civique aussi bien que morale " (Préface). Le versant civique est aussi cultivé à travers la lecture de textes historiques ${ }^{18}$. On trouve également quelques sujets témoignant de préoccupations morales: «Un peureux (ou peureuse). Ne dites pas son nom: mais vous raconterez dans quelles circonstances il (elle ) a eu peur: et vous ferez en sorte qu'il (qu'elle) se reconnaisse en lisant votre devoir» (p. 146)

Le manuel de Jean Palmero et Alexis Felix (1956) se décline en thèmes de rédaction proches de l'univers socio-culturel de l'enfant français de cette période qu'il n'invite pas à dépasser: la nature, les lieux, les activités et les métiers qui l'environnent, le temps qui passe. Comme le préconisent les auteurs de l'ouvrage, il convient d'observer le réel pour le reproduire...

On pourrait croire le manuel de René Galichet et Fernand Duplouy (1969) libéré du poids de l'idéologie. Mais certains sujets montrent que la rédaction reste un vecteur de reproduction sociale voire un facteur encourageant à la discrimination. Le lecteur moderne ne peut quêtre interpelé en songeant que les sujets suivants ont été donnés aux élèves en 1969:

Décrivez votre maman en train de préparer un repas: insistez sur sa dextérité.

Décrivez votre père lisant le journal: il est très absorbé par sa lecture et n'entend rien ni ne voit rien de ce qui se passe autour de lui.

Décrivez des romanichels dans leur campement: agitation laborieuse des femmes, paresse des hommes, jeux stupides des enfants. (p. 103)

L'écriture conserve sa vocation sociale pratique puisqu'on y rédige encore des articles, annonces, télégrammes, rapports, lettres familiales et commerciales.

18 Ces auteurs ne sont pas les seuls à procéder ainsi, la « circulaire sur les épreuves écrites du Certificat d'études primaires " du 13 mai 1946 constatant à l'époque que " la quasi totalité des thèmes de la rédaction se distribue entre les souvenirs inspirés par la guerre, la libération, les scènes de la vie familiale ou scolaire, du milieu rural ou urbain, les travaux et les travailleurs, la nature et les bêtes, les loisirs, les perspectives et les projets d'avenir ». 
Linha D’Água (Online), São Paulo, v. 28, n. 2, p. 105-121, dez 2015

\section{Des objets d'enseignement au cadre contraignant}

On sait que l'action d'enseignement se modifie profondément en fonction des caractéristiques des moyens choisis (PLANE, 1999, p. 108). En tant qu'outils guidant le maître, on s'attend à ce que ces manuels proposent des progressions, des "méthodes » d'enseignement, et qu'ils favorisent la liberté pédagogique de leur utilisateur.

Le manuel de 1947 a une organisation générale figée et répétitive. Il est difficile d'y voir une véritable progression concernant la rédaction. Le choix des textes n'est pas spécifiquement opéré en fonction des objectifs d'écriture; l'ouvrage ne fait pas de la rédaction un véritable objet d'apprentissage autonome. De par son déroulement thématique chronologique, le manuel est de nature à conditionner la pratique de l'enseignant qui peut se laisser porter au fil des saisons par cette succession de textes et d'exercices. Il laisse toutefois un peu de liberté; le choix entre plusieurs exercices au sein de chaque partie et, en y regardant de près, on découvre des activités ludiques et même un travail collectif qui peuvent permettre de différencier les apprentissages. Quant aux choix pédagogiques, nous l'avons dit, ils sont clairement classiques. A travers l'architecture du manuel persistent des présupposés théoriques que les utilisateurs, consciemment ou implicitement, ne peuvent que partager:

- La « règle de successivité» (CHERVEL, 2008) qui fait précéder à l'écriture l'étude de la langue et des auteurs. Chaque chapitre comprend des exercices de rédaction mais ils sont intégrés à la partie «Vocabulaire-Élocution » comme si les élèves nétaient pas encore prêts à écrire plus librement et amplement. L'étude des Auteurs détermine la démarche d'apprentissage.

- Lélocution et la rédaction sont considérées comme liées. En cela le manuel ne se démarque pas des préconisations institutionnelles car ces deux exercices sont censés poursuivre un objectif commun: «Apprendre à parler et écrire c'est apprendre à analyser et à traduire sa pensée »: ils « constituent au fond une même discipline " (Nouveau plan d'études des écoles primaires du 5 mars 1942)

- Une conception littéraire du texte: le texte reste sacralisé comme l'œuvre d'un écrivain « inspiré. » Il n'est pas envisagé comme le résultat d'un processus de rédaction.

Le manuel de 1956 a une organisation fragmentée: d'un côté, l'enseignement du paragraphe et une démarche qui part de l'observation, de l'autre, celui du résumé de lecture et une démarche qui part du texte. Dans chacune des deux parties, le déroulement des leçons est toujours le même et extrêmement balisé. A l'intérieur de chaque leçon, le cadre est rigoureux. La première partie (150 pages), concernant 
la rédaction d'un paragraphe, ne suit pas une progression pédagogique mais balaie " 29 centres de recherches » tirés de l'observation de la vie quotidienne ${ }^{19}$. Le maître peut donc suivre ou non cette proposition thématique, mais là encore il peut se laisser porter par cette construction. La seconde partie (30 pages), consacrée au résumé de lecture, offre en revanche une progression; résumer des mots en groupes de mots, des parties de texte en titres etc. Sous le titre « Remarquons " figure la synthèse de séances. Le maître ne peut que suivre l'ordre des leçons. Quant aux logiques d'apprentissage, ce manuel a l'avantage de pouvoir contenter les fervents de la démarche d'observation et les férus des textes d'Auteurs.

On pourrait s'attendre à ce que le manuel de René Galichet et Fernand Duplou offre aux enseignants de 1969 plus de liberté de circulation dans l'ouvrage, mais il n'en est rien. Le maître est invité à « suivre pas à pas cet ouvrage à raison d'un chapitre par semaine ». Les points abordés dans la partie méthodologique montrent, en effet, une progression logique auquel l'enseignant se doit d'adhérer (comprendre le sujet, dégager les idées d'un texte, choisir les idées, les ordonner, rédiger l'introduction, relier les paragraphes, conclure...).

Dans l'ensemble les trois manuels sont donc directifs de par leur organisation et contraignent l'enseignant à adhérer à des présupposés théoriques fondés sur le socle de l'enseignement de la rédaction: l'imitation et l'observation.

\section{Des objets d'apprentissage sous tensions}

Pour les écoliers, ces manuels sont des points d'appui à l'apprentissage, à travers des compétences attendues, à une époque où les apprenants sont confrontés à un environnement scolaire dans lequel l'écrit normé est omniprésent. Mais les logiques d'apprentissage sollicitées par ces manuels sont-elles de nature à forger des habilités rédactionnelles pour tous?

Le manuel de 1947 parie sur une démarche d'automaticité. Lélève est supposé établir de lui-même des liens entre:

19 Des lieux (ex: les rues de la ville, p. 1); des activités (ex: « les automobiles filent », p. 8), le temps (ex: la fin des beaux jours, p. 16); des métiers (ex: la travail des artisans, p. 66); la nature (ex: les oiseaux, p. 118); des sentiments (ex: la tristesse, p. 129). Les observations concernent la vue (" voir "), l'ouie (" entendre »). Les élèves doivent « comparer » (ex: « les automobiles au chemin de fer », p.6). Il s'agit aussi $d^{\prime}$ « enquêter » (ex: les espèces d'arbres, p. 121) 
Linha D’Água (Online), São Paulo, v. 28, n. 2, p. 105-121, dez 2015

\section{La lecture et l'écriture}

Lélève doit lire à haute-voix et répondre à des questions sur un texte. Ce texte est supposé être un laboratoire où s'exhibent des procédés d'écriture. L'imitation permet une reprise de certains matériaux, mais les spécificités de chaque texte ne sont pas montrées. L'articulation texte/écriture ne se fait que par une activité mécanique de reproduction («Imitons ») ou de réduction du texte («Enchaînons les idées »).

\section{La langue et l'écriture}

L'écriture est conçue comme une procédure d'intégration assez aléatoire de matériaux issus d'autres activités au sein de sous-disciplines créées par démembrement du Français (vocabulaire, orthographe, grammaire, lecture...). Il n'y a pas d'invitation à la manipulation ou à la transformation. Il n'y a pas de travail sur la réécriture visant l'amélioration du texte. Lécriture reste une activité périphérique à la langue et construite depuis son observation.

Les thèmes de rédaction d'un paragraphe sont majoritairement proches de l'univers social de l'enfant: «Autour de la table, nous écossons les haricots » (p.12); «Veille charrette ; vieux cheval» (p. 189)

La rédaction est souvent facilitée en apparence car guidée par un « calque » (texte à trous) ou des questions largement orientées:

Je cueille (ou : je voudrais cueillir) de beaux raisins. Je prends...(Quoi ?) - Je vais (Où ? dans la vigne ? au pied de la treille ?) - Je regarde les fruits (Comme ils sont beaux et appétissants !) - Je ... (Que faites-vous ? Prenez-vous une échelle ? Quels fruits choisissez-vous ? Comment les coupez-vous ? Avec précaution ? ou en vous hâtant ?) Je rentre en... (Vous goûtez sans doute quelques grains). (p. 12)

La question est de savoir si tous les exercices sont vraiment de nature à développer des habilités scripturales tant l'écriture est conditionnée. L'élève n'a souvent le choix ni des idées ni même des mots.

Voici un exercice:

J'aime (ou je n'aime pas) ma salle de classe. Ma salle de classe est une pièce... (spacieuse - exiguë - rectangulaire, carrée, presque carée, de ... mètres de long sur ...mètres de large). Elle est éclairée par de...(grandes, petites fenêtres, qui donnent sur...). Les tables sont... (commodes, incommodes. Est-on bien ou mal assis ?). 


\section{Linha D’Água (Online), São Paulo, v. 28, n. 2, p. 105-121, dez 2015}

Les murs sont.... (blanchis de neuf, d'un gris sale, couverte de poussière) et (bien décoré de...ou tristement recouverts de...). (p 12)

Lélève peut difficilement rédigé autrement que:

J'aime ma salle de classe. Ma salle de classe est une pièce spacieuse presque carée de ... mètres de long sur ...mètres de large. Elle est éclairée par de grandes fenêtres qui donnent sur.... Les tables sont commodes. On est bien assis. Les murs sont blanchis de neuf et bien décoré de...

Le manuel de 1956 part de la démarche d'observation qui s'ajoute à celle de l'imitation. L'écolier doit mettre en forme les observations qu'il a rédigées dans ses " fiches d'observation $»^{20}$ en de « courts instantanés ». Il est censé savoir produire un écrit à l'issue d'un processus « entonnoir » qui part de l'observation du réel, se structure grâce aux temps de vocabulaire ${ }^{21}$ et d'élocution et s'embellit de l'étude des textes, le tout pour devenir un paragraphe. Ce processus est présenté comme quasi « naturel »:

Si vous avez bien rempli votre fiche d'observations, il vous sera facile de rédiger de beaux récits. A votre âge, pour être bon écrivain, il suffit d'avoir de bons yeux; Vous allez le comprendre en complétant (d'après vos observations) les phrases suivantes... (p. 3)

Remarquons: Les auteurs de beaux textes n'emploient pas n'importe quel verbe. Ils choisissent celui qui montre le mieux l'action d'une personne, d'un animal ou d'une chose. Essayons de les imiter (p. 24)

$\grave{A}$ votre tour en vous inspirant de ce bel exemple et en vous souvenant de vos observations, écrivez un court et précis instantané sur un ou plusieurs des sujets suivants... (p. 5)

Il n'existe pas de réflexion sur deux points fondamentaux: comment l'élève mémorise- $\mathrm{t}-\mathrm{il}$ toutes ces informations et comment les articule- $\mathrm{t}-\mathrm{il}$ pour rédiger ?

On note toutefois quelques points de nature à aider les scripteurs:

20 L'élève est invité, en dehors des heures de classe, à remplir une « fiche d'observations » en répondant à des questions, en enquêtant, en comparant.

${ }^{21}$ La leçon de vocabulaire doit permettre de préciser (" effort d'exactitude ») et de justifier (" effort de sincérité ») le sens des mots utilisés. 
Linha D’Água (Online), São Paulo, v. 28, n. 2, p. 105-121, dez 2015

- Le recours aux images (technique venue des Etats-Unis selon CHERVEL, 2008) ${ }^{22}$ qui offre une entrée supplémentaire vers l'écrit et peut-être plus concrète pour certains écoliers.

- Une proposition pour se confronter aux performances des autres apprenants. En effet, la correction est collective. Le meilleur « instantané » de la classe est choisi (sans doute par le maître ?) et consigné au tableau, puis critiqué oralement. Chaque élève doit ensuite rechercher individuellement une précision ou une expression « plus parfaite » lesquelles, est-il précisé, « peuvent se trouver dans son propre instantané ».

- Une approche métacognitive: chacune des parties comporte sous le titre "Remarquons » des temps de synthèse sur ce qui est mis en exergue du point de vue de l'apprentissage:

Un paragraphe est un ensemble de phrases se rapportant à une même idée principale. Dans un récit comportant une suite d'idées différentes, il y a autant de paragraphes que d'idées principales (p.21)

La lecture du texte achevée, je dois pouvoir écrire la suite des titres qui résument chacune des parties du fragment. Je supprime les titres inutiles : ce qui reste constitue le plan du récit que j’ai à résumer (p. 167)

Ce mouvement réflexif sur les procédures d'écriture n'est peut être pas encore construit par l'élève lui-même mais il a le mérite d'exister. (PLANE, 1999, p. 131)

- Enfin, on trouve dans la seconde partie de l'ouvrage, une approche du genre puisque les auteurs ont voulu des textes « de genres variés ». Cette diversification est un facteur de motivation des élèves (PLANE, 1999, p. 138) et l'on sait également que la construction de la notion de genre facilite la rédaction (CRINON, 2006).

Cependant, ces deux manuels se heurtent à deux écueils. D'un côté, les auteurs (surtout ceux du manuel de 1947) déclarent vouloir confronter les élèves aux œuvres littéraires pour mieux écrire, mais les clés de lecture et de réinvestissement de ces textes ne sont pas mises en évidence. Or, il ne suffit pas de mettre les élèves en présence d’objets culturels pour combler les inégalités socio-culturelles

22 Le manuel propose la description de dessins et invite à les comparer avec la réalité. On sait que Buisson et Berger ont rapporté des Etats Unis un exercice élémentaire « la rédaction sur image » et que les adversaires de cette méthode ont préconisé de lui préférer l'observation du réel. (CHERVEL, 2008) 
(BONNERY, 2007). De l'autre côté, certains exercices, plus conformes aux exigences institutionnelles, partent de l'observation du réel ou de l'expérience personnelle de l'enfant, mais il a été montré que la transposition du vécu ordinaire et intime de l'enfant en connaissance dépersonnalisée n'est pas automatique (BONNERY, 2007). Comment apprendre à formuler cette expérience et ce ressenti en dehors des « béquilles » de l'imitation de phrases ? Il ne suffit pas de voir, de ressentir, et de se rappeler, pour savoir écrire.

Dans le manuel de 1969, le rapport au texte est désacralisé au profit d'une approche exploratoire de l'écrit que l'on peut transformer ou poursuivre. Les écrits à produire sont plus variés et recouvrent une approche plus complète du récit (décrire un ensemble/des détails/un animal/un personnage immobile/un personnage en action) même s'ils restent centrés sur la vie personnelle, pratique ou professionnelle. L'élève se trouve toujours investi dans la démarche de préparation (observation du réel, enquête) mais le dispositif est moins lourd qu'en 1956 avec la « fiche d'observation ». La partie méthodologique permet de rendre plus visible ce qu'on attend du scripteur et institutionnalise quelques savoirs ou conseils. Il s'agit désormais de s'approprier des méthodes de rédaction transférables à d'autres situations d'écriture. Louvrage est ambitieux tant au niveau du nombre de points méthodologiques abordés (vingt-deux) que de la variété des écrits à produire ${ }^{23}$. Il n'est donc pas certain qu'il laisse un temps suffisant à chaque apprentissage.

\section{Conclusion}

Ces trois manuels renseignent en défnitive sur le statut des savoirs, les modes d'enseignement, les modes d'apprentissage et les finalités de l'école dans cette période d'aprèsguerre d'une France encore majoritairement rurale et conservatrice. Ces ouvrages témoignent de la résistance de la communauté éducative aux textes réglementaires quand ils préconisent le recours aux textes littéraires et à l'imitation pour écrire de « belles phrases ». Ils montrent, toutefois, l'abandon progressif de l'hégémonie de l'imitation issue du modèle porté par l'enseignement du Secondaire. Il s'opère en effet progressivement un glissement des pratiques, de l'imitation des textes et de l'observation du réel vers une démarche plus expérimentale et méthodologique, même si tout semble planifier. L'analyse nous révèle aussi que les enseignants sont dirigés par des manuels définissant un cadre majoritairement contraignant. Ces

23 A côté des récits de vie persistent des sujets pratiques ou professionnels (rédiger un article, une annonce, un télégramme, p. 139; un rapport, p. 168; une lettre, p. 200; une lettre commerciale, p. 207; soigner la présentation matérielle, p. 214). Mais l'on trouve aussi traités différents points permettant de construire un récit: descriptions diverses, dialogue, expression des sentiments, portrait. 
ouvrages interrogent sur les modes d'apprentissage retenus parce qu'ils n'offrent pas encore la possibilité d'inscrire l'écrit dans un projet particulier de la classe ou de l'élève, que la diversité des petits exercices non replacés dans leur finalité intellectuelle encourent le risque de faire travailler sur des « micro procédures de surface » et de ne pas construire du sens (PLANE, 1999, p. 39), et que l'élève n'a pas de "grille de contrôle " pouvant l'aider à s'évaluer ou pouvant lui servir de guide lors d'une tâche de réécriture, d'ailleurs non organisée. Il manque sans doute à ces ouvrages la réflexion sociologique tirée des enseignements de Goody selon laquelle une partie de l'échec scolaire tient au fait que l'école engage trop rapidement les apprentissages formels en faisant l'économie d'apprentissages culturels sur la raison graphique. Enfin, ces manuels portent en eux les débats sur les missions de l'école. Tout en continuant à préparer l'élève à devenir un être social et professionnel (écrits pratiques), avec l'allongement de la scolarité, la maitrise de la rédaction est présentée comme un « passeport pour la classe supérieure $»^{24}$. A travers l'enseignement de la rédaction sont encore véhiculés des notions morales et civiques qui renseignent sur les valeurs adoptées par la société. Parce que, au-delà de la maîtrise du français et de la connaissance de la littérature, on cherche à former la pensée.

\section{Références Bibliographiques}

BISHOP, Marie-France. Les annotations, indicateurs des fonctions de la rédaction: parcours historique. Repères, n. 31, p. 9-27, 2005.

BONNERY, Stéphane. Comprendre l'échec scolaire. Elèves en difficultés et dispositifs pédagogiques. La dispute, coll. « Lenjeu scolaire », 214 p., 2007. - http://lectures.revues.org/523

CHERVEL, André, l'enseignement du français à l'école primaire, tome II, 1880-1939 et tome III, 1940-1995, INRP Economica, 497 p., et 633 p., 1995.

CHERVEL, André, Histoire de l'enseignement du français du XVII au XXe siècle. Retz, 830 p., 2008.

CRINON, Jacques. Lécriture littéraire et les genres, Le Français aujourd'hui n. 153, p. 17-24, $2006 / 2$.

24 La formule est utilisée par le manuel de 1969. Y sont recherchés une place dans la société (exercices pratiques) la formation d'un honnête homme (messages moraux) le développement d'un talent esthétique (belles phrases, le style) et des habiletés pour communiquer et penser. 
Linha D’Água (Online), São Paulo, v. 28, n. 2, p. 105-121, dez 2015

GALICHET, René; DUPLOUY, Fernand. Méthode active d'initiation à la composition française par le vocabulaire et la construction de phrases, Editions Charles-Lavauzelle et Cie, p. 1-219, 1969.

PALMERO Jean; FELIX Alexis. Rédigeons, le résumé de lecture, le vocabulaire, le paragraphe par l'observation. Cours Moyen. Hachette, p. 1-186, 1956.

PIERRE ANTOINE. Doux parler de France, Lecture Langue Française, Cours Moyen. Librairie Istra, 224 p., 1947.

PLANE, Sylvie (Dir.). Manuels et enseignement du français. Actes du colloque Enseigner le français avec ou sans manuel. CNDP coll. Actes et documents. Paris: SCEREN, 1999.

\section{Textes officiels}

instructions relatives au nouveau plan d'études des écoles primaires du 5 mars 1942 Arrêté concernant les horaires et programmes des écoles primaires du 17 octobre 1945

Arrêté modifiant le règlement du certificat d'études primaires du 22 février 1946 Circulaire sur les épreuves écrites du Certificat d'études primaires du 13 mai 1946 Arrêté fixant le programme des classes de fin d'études du 25 juillet 1947 Arrêté réorganisant le certificat d'études primaires du 28 octobre 1947 Instructions sur le certificat d'études primaires du 30 octobre 1947 Circulaire sur l'organisation du certificat d'études primaires du 16 avril 1949 Circulaire du 17 mars 1952 relative aux conférences pédagogiques de 1952 Circulaire concernant l'enseignement du français et du calcul dans les classes primaires du 19 octobre 1960 